\title{
Control of the Lateral Displacement Restoring Force of IRWs for Sharp Curved Driving
}

\author{
Hanwoong Ahn*, Hyungwoo Lee**, Sungchul Go***, Yonho Cho ${ }^{\S}$ and Ju Lee ${ }^{\dagger}$
}

\begin{abstract}
This paper presents a lateral displacement restoring force control for the independently rotating wheelsets (IRWs) of shallow-depth subway systems. In the case of the near surface transit, which has recently been introduced, sharp curved driving performance is required for the city center service. It is possible to decrease the curve radius and to improve the performance of the straight running with the individual torque control. Therefore, the individual torque control performance of the motor is the most important point of the near surface transit. This paper deals with a lateral displacement restoring force control for sharp curved driving. The validity and usefulness of the proposed control algorithm is verified by experimental results using a small-scale bogie system.
\end{abstract}

Keywords: Individual torque control, IRW, Lateral displacement restoring force, Shallow-depth subway systems

\section{Introduction}

For the existing high-depth subway systems, the construction fee of the operator increases. Moreover, the transfer distance and time with the existing subway also increase aggravating social cost and inconvenience of users. The diversification of transportation due to the development of cities and transportation situation focuses on shallow-depth subway systems through maximized space, excellent accessibility, and environment-friendly/ future-style transportation. [1-2]

For the fixed wheelsets that are generally applied to trains, the axis is assembled where squeal noise can occur along with difficult accessibility for passengers. When using sharp curved driving technology of independently rotating wheelsets, curve radius of $15 \mathrm{mR}$ dejected drive is possible within the urban. Furthermore, the construction fee that occurs in accordance to the limitation of the radius of curve can be reduced, and the construction period can also be reduced. Thus, lateral restoring force control for sharp curve drive of independently rotating wheel suspension is the core technology of the low-depth urban railway system. [3-7]

In this paper, a control of the lateral displacement restoring force for sharp curved drive of the shallow-depth subway systems is proposed. The drive torque, steering

$\dagger$ Corresponding Author: Dept. of Electrical Engineering, Hanyang University, Korea. (julee@hanyang.ac.kr)

* Dept. of Electrical Engineering, Hanyang University, Korea. (zemlja@naver.com)

** Dept. of Railway Vehicle System Engineering, Korea National University of Transportation, Korea. (krhwlee@ut.ac.kr)

*** Mechatronics R\&D Center, Samsung Electronics Co., Korea. (sc.go@samsung.com)

$\S \quad$ Bogie Development Team, Hyundai Rotem Co., Korea. (ycho@hyundai-rotem.co.kr)

Received: August 24, 2015; Accepted: February 1, 2016 torque command were made to execute this research to increase the stable drive performance without contacting the flange in the curved-railroad. Also, a small-scale bogie system was copied from the shallow-depth subway systems for the independently rotating wheelsets to verify the performance of the proposed lateral restoring force control.

\section{Small-scale Bogie System}

The electrical analysis obtained through theory and simulation of the proposed lateral restoring force control is meaningless in the real system because electrical systems and mechanical systems are closely coupled. Therefore, validation of the proposed algorithm was made through the actual production. Moreover, to verify the validity of lateral restoring force control, the actual size of the lowdepth suspension must be produced. However, for the acquisition of lines and production of urban low-depth suspension, many time and investment are needed. Thus, $1 / 5$ scale law of similarity as shown in Table 1 was used to design and make the small-scale bogie system.

The designed small-scale independent rotating wheelsets system is shown in Fig. 1. Fig. 1(a) shows the small-scale

Table 1. Physical quantities according with the law of similarity

\begin{tabular}{c|c|c|c}
\hline Scale Factor & Scale Law & Scale Factor & Scale Law \\
\hline Length & $1 / 5$ & Inertia & $1 / 5^{5}$ \\
\hline Time & 1 & Elasticity & 1 \\
\hline Frequency & 1 & Weight & $1 / 5^{3}$ \\
\hline Velocity & $1 / 5$ & Force & $1 / 5^{4}$ \\
\hline Acceleration & $1 / 5$ & Stiffness & $1 / 5^{3}$ \\
\hline Density & 1 & Damping & $1 / 5^{3}$ \\
\hline Mass & $1 / 5^{3}$ & Friction & 1
\end{tabular}


bogie that is composed of 4 permanent-magnet synchronous motors. Each motor is in charge of one wheel, and the input voltage of the motor is supplied through different inverters. There is 6:1 reducer installed between the wheel and the motor that increases the torque of the small-scaled independently rotating wheelsets system. The primary suspension system part of the small-scale bogie was designed to move from left to right. So, the small-scale bogie was designed as a structure to create lateral force. Also, laser sensors were attached to the center party of the suspension system so that it can sense lateral displacement. Fig. 1(b) shows small-scale roller rig. The roller rig substitutes the rail, which was designed to compose the test environment for the small-scale bogie to operate. The wheel of the small-scale bogie and the rail wheel of the roller rig are in contact for operation. Furthermore, the small-scale roller rig's motor was composed so that the rail wheel can rotate left and right to implement the load status according to the slope of the small-scale bogie.

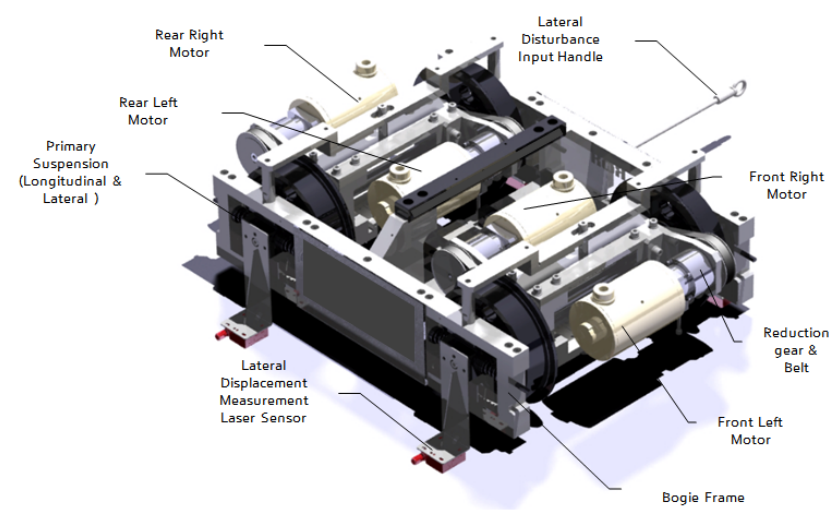

(a) Small-scale bogie

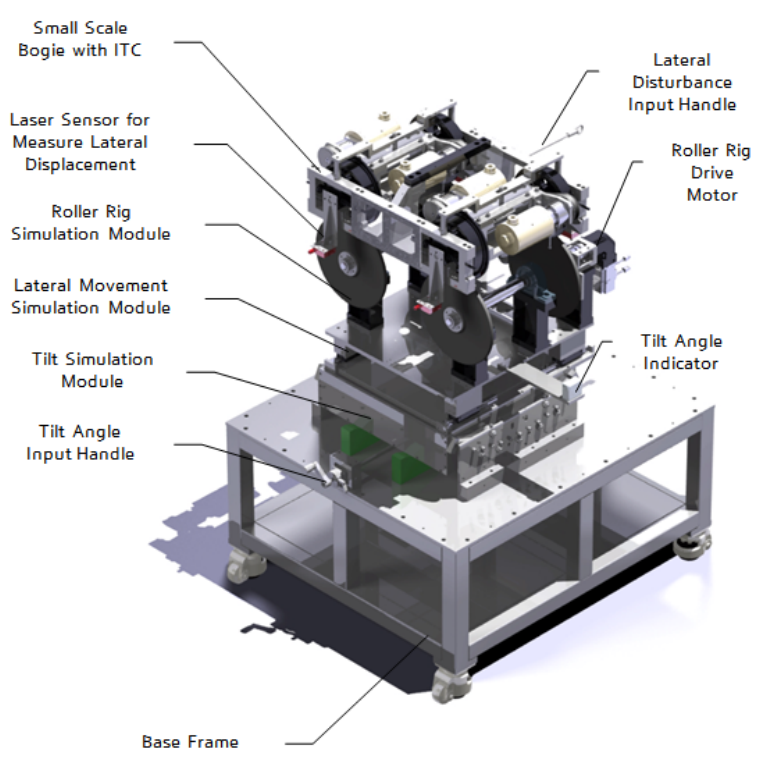

(b) Small-scale roller rig

Fig 1. Small-scale independent rotating wheelsets system

\section{The Characteristics of Independent-wheel Type Motors}

The size of the motor for the small-scale bogie is $1 / 5$ scale of the actual urban low-depth train. Thus, the load torque is decided according to (1) in the base speed of small-scale motor.

$$
T_{m}=\left(\frac{\pi}{4} k_{w} \hat{B}_{g 1} \cos \beta\right) D_{g}^{2} L_{s t k}
$$

where, $k_{w}$ : the winding factor, $\hat{B}_{g 1}$ : the peak of the fundamental wave of the air-gap magnetic density, $a c$ : the ratio of electric loading, $\beta$ : the current phase angle, $D_{g}$ : the diameter of air-gap, and $L_{s t k}:$ the length of axial.

For the small-scale motor, the length of each side is reduced by $1 / 5$ where the total volume becomes $1 / 125$. The output by (1) becomes $1 / 125$, thus $360 \mathrm{~W}$. This paper considered the output margin to design $500 \mathrm{~W}$ motor. Thus, when $15[\mathrm{~km} / \mathrm{h}]$ base speed is selected as sharp curve drive speed for the test of small-scale bogie, the load torque value in base speed in $2[\mathrm{Nm}]$.

The motor of independently rotating wheelsets selected model in accordance to performance of control to independently control 4 motors. Magnetic torque and reluctance torque are the two torques that exist in the motor. In the case of reluctance torque, it occurs due to interaction

Table 2. The specification of the motor of independently rotating wheelsets

\begin{tabular}{c|c|c}
\hline Parameter & Value & Unit \\
\hline Poles/Slots & $10 / 12$ & $\mathrm{~mm}$ \\
\hline Outer Diameter & 80 & $\mathrm{~mm}$ \\
\hline Stack & 60 & $\mathrm{~V}$ \\
\hline $\mathrm{V}_{\mathrm{dc}}$ & 48 & $\mathrm{~A}_{\text {peak }}$ \\
\hline $\mathrm{I}_{\mathrm{am}}$ & 10 & $\mathrm{Ohm}$ \\
\hline $\mathrm{R}_{\mathrm{a}}$ & 0.06 & $\mu \mathrm{H}$ \\
\hline $\mathrm{L}_{\mathrm{s}}$ & 328.5 & $\mathrm{mWb}$ \\
\hline$\Phi_{\mathrm{a}}$ & 18.6 & $\mathrm{RPM}$ \\
\hline$\omega_{\text {base }}$ & 2400 & $\mathrm{Nm}$ \\
\hline $\mathrm{T}_{\max }\left(@ \omega_{\text {base }}\right)$ & 2.26 &
\end{tabular}

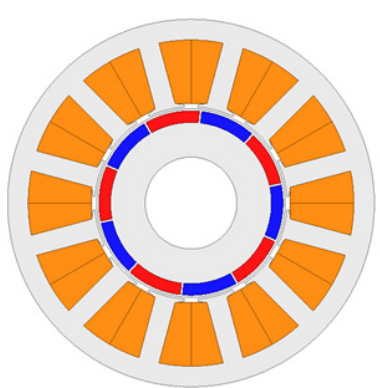

Fig. 2. The model of the motor of independently rotating wheelsets 
of d-axis and q-axis currents. The controllability reduces compared to the magnetic torque that only uses d-axis current. Thus, the motor was designed with SPMSM (Surface Permanent Magnet Synchronous Motor) using only the magnetic torque. [8-9]

For SPMSM, CAN structure exists to prevent permanentmagnetic scattering when rotating. Due to this, there is a disadvantage of increased length of opening. To supplement the weakness of this rotor, the shape as shown in Fig. 2 was selected. The shape was similar to IPMSM (Interior Permanent Magnet Synchronous Motor), but the magnetic flux density on the rib and web part is saturated where the inductance values of d-axis and q-axis are the same. Thus, it has the same characteristic as SPMSM that only has the magnetic torque.

The independent wheel motor was designed as shown in

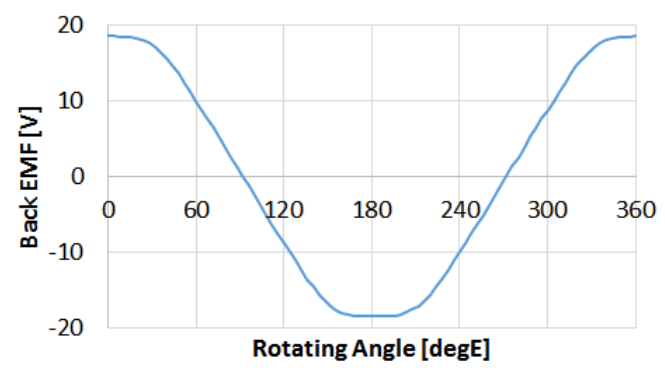

(a) Back EMF

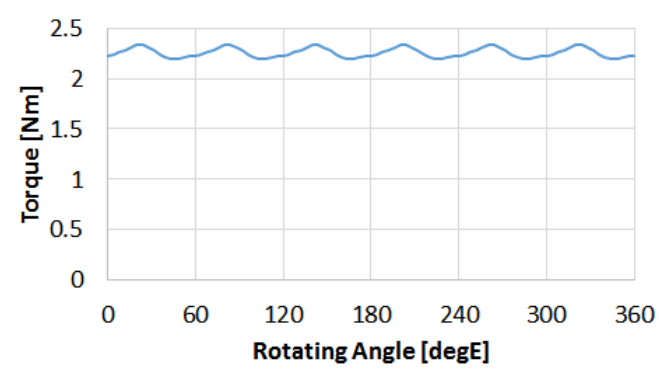

(b) Electrical Torque

Fig. 3. Analysis Result of the independent wheel motor at rated speed
Table 2, and Fig. 3 shows the analysis result of rotation in 2400 [RPM] for the independent wheel motor. No-load phase EMF waveform was verified to be sinusoidal, and the mean value of torque was 2.26 [Nm], which satisfied the required torque $2[\mathrm{Nm}]$.

\section{The Control of the Lateral Displacement Restoring Force}

\subsection{The torque control of independent wheel motor}

The motor applied to the produced small-scale bogie was SPMSM. When adding the proposed lateral restoring force control to SPMSM's torque control system, torque control block of independent wheel motor can be composed like Fig. 4. The PMSM control algorithm in Fig. 4 is the control block corresponding to 1 motor. When 3 parallel control blocks are added, the complete control block of the independently rotating wheel motor can be obtained. To acquire the power of small-scale bogie, torque reference $T_{c}$ is produced to the 4 SPMSM. Here, the lateral restoring torque reference $T_{l}$ is added to the 4 motor creating the motor to create torque reference $T_{e}$. The torque reference is changed to $\mathrm{d}$-axis and $\mathrm{q}$-axis current reference from the torque constant, and the voltage reference is formed by passing the PI current controller. The voltage reference is decides the switching status of the three phase inverter through inverse d-q transformation, and the voltage is induced to the SPMSM creating the torque desired by the motor. Here, for the d-q transformation and inverse $\mathrm{d}-\mathrm{q}$ transformation, a position sensor is needed. Thus, the lateral displacement is recognized as the laser sensor creating $T_{l}$ by comparing the initial status.

\subsection{The control of the lateral displacement restoring force of the IRWs}

Fig. 5 shows the drive of the straight-railroad and curved-railroad of independently rotating wheelsets. The

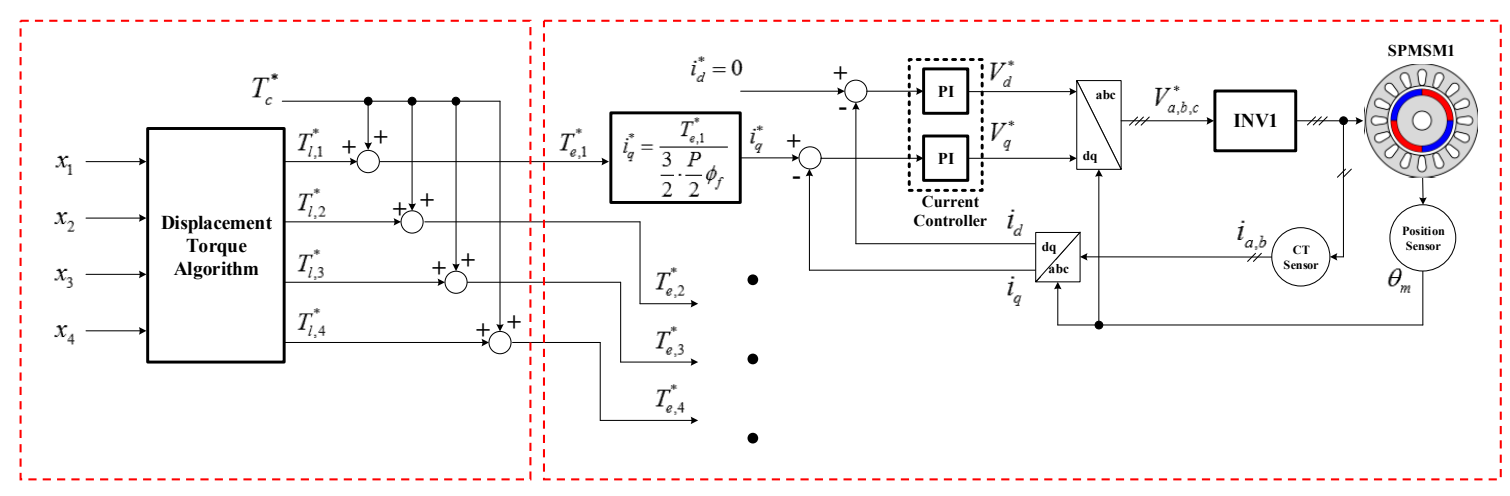

Lateral Restoring Control

PMSM Torque Control

Fig. 4. SPMSM torque control system using lateral restoring control 


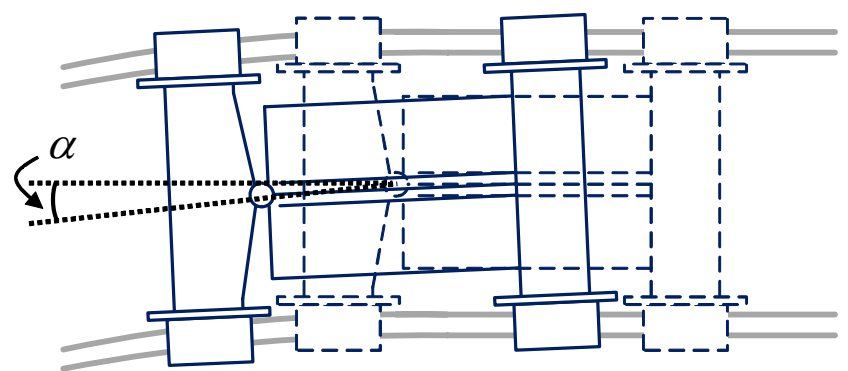

Fig 5. Curved-railroad of independently rotating wheelsets

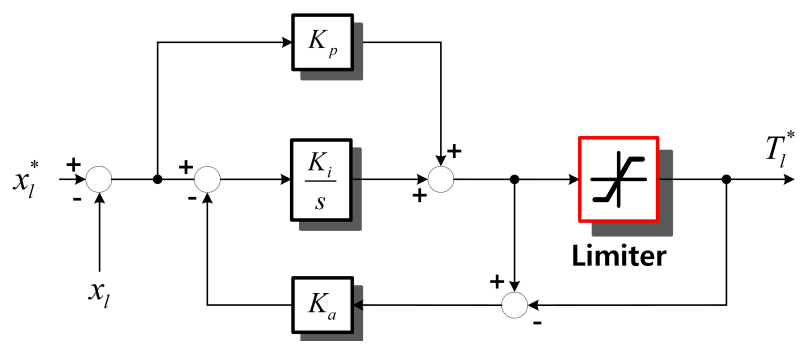

Fig. 6. Lateral restoring controller

dotted line shows the straight-railroad, and the solid line shows the curved-railroad. When charging into the curved-railroad and recognizing this through the lateral displacement sensor, lateral force must occur to recover attack angle $\alpha$ to avoid flange contact. Hereby, steering torque of each motor is required, and then $T_{l}$ is created through the lateral recovering controller like Fig. 6. The lateral recovering controller commands the initial location of independently rotating wheelsets, and then receives feedback on the location of the bogie through lateral displacement sensor. Output value $T_{l}$ acquired through PI controller is shown in the following (2). Here, the maximum value and minimum value of output $T_{l}$ must be less than the drive torque of suspension so that reverse torque does not occur. Thus, the limiter values were set to $90 \%$ of $T_{c}$.

$$
T_{l}=\left(K_{p}+\frac{K_{i}}{s}\right) E(s)
$$

Here, $E(s)$ represents lateral displacement, which shows the error between the location of the bogie when operating and the location of bogie initially.

The independently rotating wheelsets can independently control 4 wheels creating different lateral restoring torques according to the location of the bogie. When the drive of bogie is not considered, and when lateral displacement occurs to the left like the dotted shape of Fig. 7(a), the torque on the motor corresponding to the left of the front and rear wheels shall be made for recovery to initial location to create lateral force to the right. Thus, in this case the bogie can be recovered to the initial location like the solid line. However, this is the same as pushing the

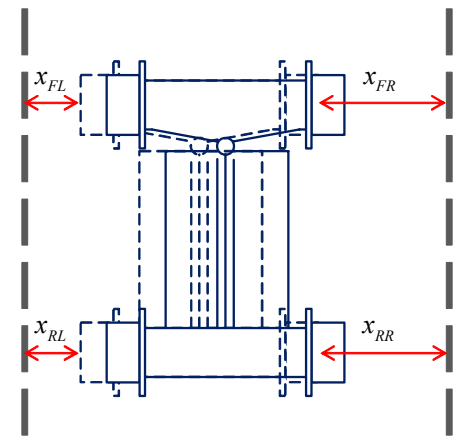

(a) Same Direction

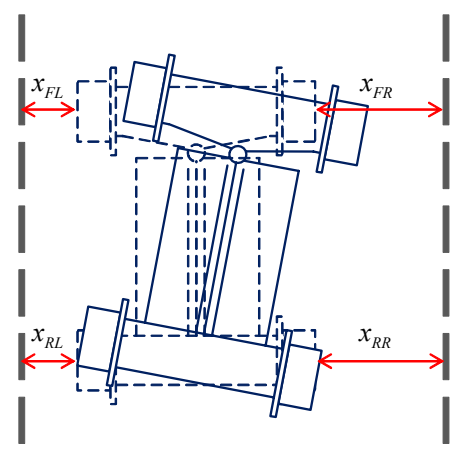

(b) Opposite Direction

Fig. 7. The independently rotating wheelsets in accordance with the lateral restoring torque methods

bogie towards the left. Thus, the drive torque cannot be used needing a lot of recovery power, and in the worst case, recovery can be impossible. Fig. 7(b) shows the case of giving lateral restoring torque like (3).

$$
\left\{\begin{array}{l}
\Delta x_{F R}>0 \Rightarrow T_{l, F R}>0, \quad \Delta x_{F R}<0 \Rightarrow T_{l, F R}<0 \\
\Delta x_{F L}>0 \Rightarrow T_{l, F L}>0, \quad \Delta x_{F L}<0 \Rightarrow T_{l, F L}<0 \\
\Delta x_{R R}>0 \Rightarrow T_{l, R R}<0, \quad \Delta x_{F R}<0 \Rightarrow T_{l, R R}>0 \\
\Delta x_{R L}>0 \Rightarrow T_{l, R L}<0, \quad \Delta x_{F R}<0 \Rightarrow T_{l, R L}>0
\end{array}\right.
$$

As shown in Fig. 7(b), when the bogie drifts to the left, the front wheel makes the torque to the motor that corresponds to the left, and the rear wheel makes the torque to the motor corresponding to the right. Then, the suspension moves along with the solid line, and the bogie moves right due to the drive torque. Thus, because the drive torque is used, relatively small steering torque can be used for later restoring torque, and the response is also increased. This paper proposes mechanism like this, and verified the lateral restoring torque of independently rotating wheelsets.

\section{Experimental Results}

The small-scale bogie was made as shown in Fig. 8(a) by using 4 independent wheel motors. Each motor was in 
charge of the each bogie's wheel, and it was connected through the reducer. The rotor position of the motor used encoder for measurement. To recognize the lateral displacement of small-scale bogie, 4 laser sensors were fixed on the center part of the wheel.

Fig. 8(b) used independent wheel motor to show the later recovering control test inverter and controller. Because of the $1 \mathrm{C} 1 \mathrm{M}$ system, there are 4 motors. DSP used

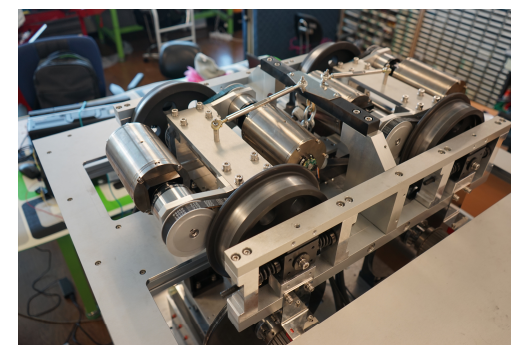

(a) Small-scale bogie

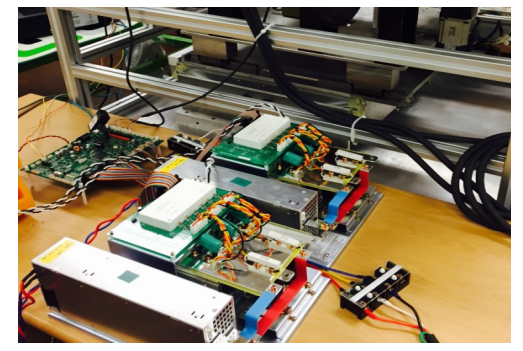

(b) DSP and inverters

Fig. 8. Experimental test setup

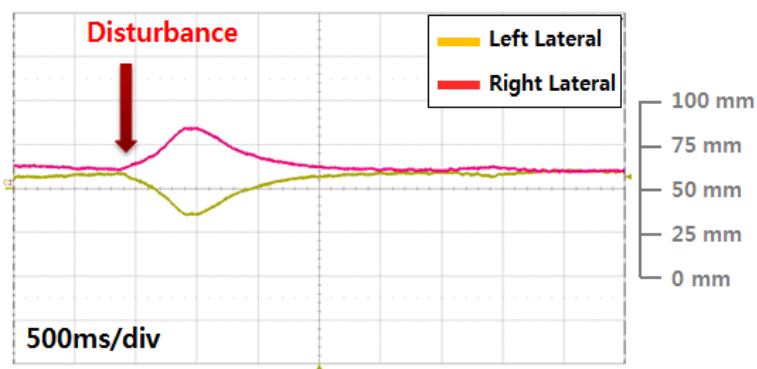

(a) Lateral displacement of the front wheel

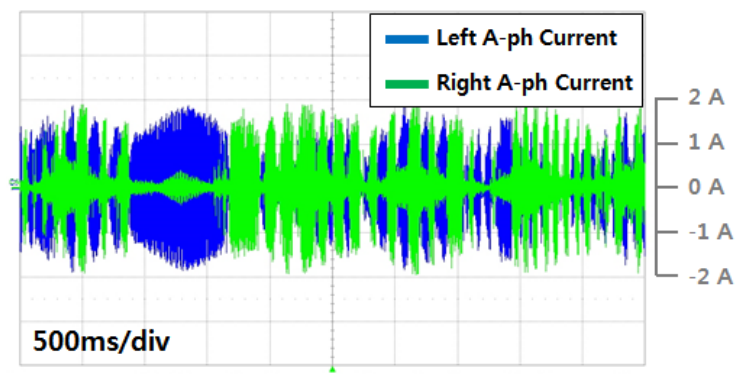

(c) A-phase current of the front wheel
TI28335, and because 12 switching signals can be created per 1 DPS, a total of 2 DSPs were used.

The result of lateral recovering control test using smallscale bogie is shown in Fig. 9. Fig. 9(a), (b) respectively identified the lateral displacement of the front and rear wheels of the small-scale bogie, and it is the output obtained through the laser sensor. When the right and left lateral displacements are same, the small-scale bogie does not lean towards one side being able to maintain centering. Thus, it was represented that centering was maintained before disturbance occurred. Also, it was shown that recovery is possible even when disturbance occurs. Thus, the validity of the proposed lateral recovering control algorism was confirmed. Fig. 9(c), (d) respectively shows the A phase current of the small-scale independent wheel motor. When disturbance does not occur, it was identified that the magnitude of current of the left and right motors change to maintain centering. Also, when the disturbance occurs, one side created greater torque to recover lateral displacement. Because the lateral displacement occurred close to the right side, the front wheel according to (3) showed increasing torque of right motor, and the rear wheel showed increasing torque of the left side motor. This could be shown in the Fig. 9(c), (d).

\section{Conclusion}

During sharp curve drive according to the characteristic of the train, noise is frequently induced due to the contact of flange. This is because 4 motors are controlled by the

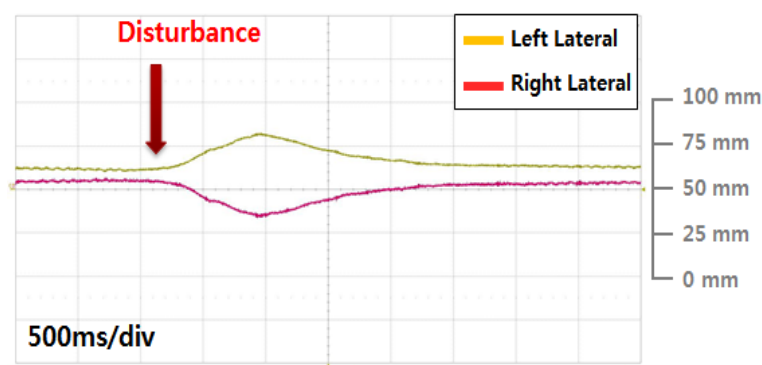

(b) Lateral displacement of the rear wheel

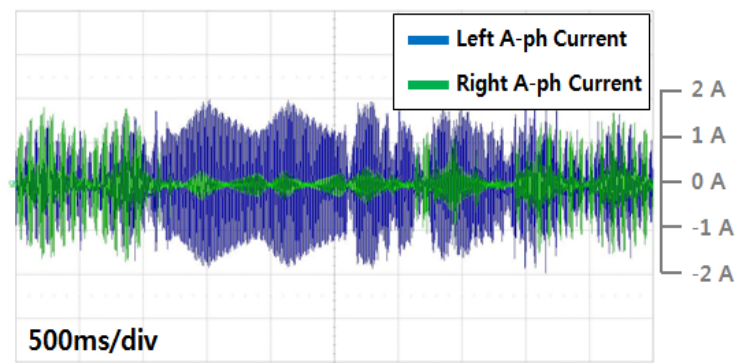

(d) A-phase current of the rear wheel

Fig. 9. Test result of lateral restoring control 
same torques in the $1 \mathrm{C} 4 \mathrm{M}$ system where the flange contacts the rail for the curved-railroad. Furthermore, this induces unpleasant noises causing abrasion. Thus, the curve radius of the suspension becomes limited. This paper introduced $1 \mathrm{C} 1 \mathrm{M}$ system using the difference of each motor torque to create lateral force proposing the algorism to avoid flange contact to solve this problem.

For the permanent magnetic synchronous motor, the torque ripple is smaller compared to the induction motor. Also, with the advantage of high torque density, it is receiving spotlight as the future traction motor. This paper designed and made SPMSM (Surface Permanent Magnet Synchronous Motor), and also applied 1/5 scale for the small-scale bogie to verify the later recovering control algorism.

Lateral displacement was measured by using laser sensor, and different torque commands were given to each motor through lateral recovering controller to avoid flange contact. When disturbance occurs in the small-scale bogie, centering can be maintained, which demonstrated the superiority of algorism. Furthermore, based on the lateral recovering algorism proposed through the small-scale bogie, it is considered that the algorism can be applied to the actual train system.

\section{Acknowledgements}

This research was supported by Basic Science Research Program through the National Research Foundation of Korea(NRF) funded by the Ministry of Science, ICT \& Future Planning(No. 2013R1A1A1061048)

\section{References}

[1] H. Glickenstein, "News and Views in Transportation", IEEE Vehicular Technology Magazine, Vol. 9, No. 2, pp. 14-19, Jun. 2014.

[2] C.Y. Chang, J.M. Kim and Y.H. Kim, "A Design of Prototype 1C2M Railway Vehicle Propulsion Control System Considering Slip Reduction of Traction Motor", Journal of Electrical Engineering \& Technology., Vol. 10, No. 1, pp. 429-435, Jan. 2015.

[3] B. Liang and S.D.Iwnicki, "An Experimental Study of Independently Rotating Wheels for Railway Vehicles", ICMA 2007, pp. 2282-2286, Aug. 2007.

[4] Roger Goodall and T X Mei, "Mechatronic strategies for controlling railway wheelsets with independently rotating wheels", in AIM 2001, pp. 225-230, Jul. 2001.

[5] T. X. Mei and Roger M. Goodall, "Robust Control for Independently Rotating Wheelsets on a Railway Vehicle Using Practical Sensors", IEEE Transactions on Control Systems Technology, Vol. 9, No. 4, pp. 599-607, Jun. 2001.
[6] A.H. Wickens, "Dynamic stability of articulated and steered railway vehicles guided by lateral displacement feedback", Vehicle Syetem Dynamics, Vol. 23 (supplement), pp. 541-553, 1994.

[7] Y. Cho, J. Kwak, "Development of a New Analytical Model for a Railway Vehicle Equipped with Independently Rotating Wheels", International Journal of Automotive Technology, Vol. 13, Np. 7, pp. 10471056, Dec. 2012.

[8] Z. Peroutka, K. Zeman, F. Krus and F. Kosta, "New generation of full low-floor trams: Control of wheel drives with permanent magnet synchronous motors", in ECCE 2009, pp. 1833-1840, Sep. 2009.

[9] Z. Peroutka and K. Zeman, "Design Considerations for Control of Traction Drive with Permanent Magnet Synchronous Machine," in EPE-PEMC 2008, pp. 1529-1534, Sep. 2008.

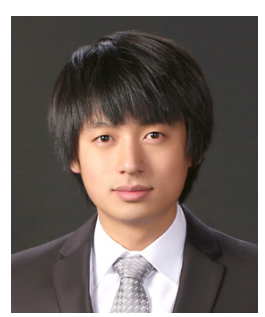

Hanwoong Ahn He received his B.S. and M.S. degrees in Electrical Engineering from Hanyang University, Seoul, Korea in 2010 and 2012, respectively. Since 2012, he has been pursuing the Ph.D. degree at the Department of Electrical Engineering, Hanyang University. His research interests include design, analysis, testing and control of motor/generator; power conversion systems; and applications of motor drive.

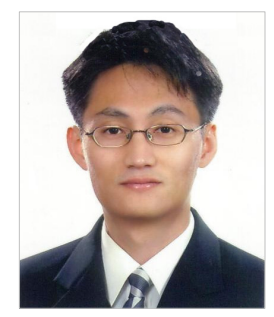

Hyungwoo Lee He received his M.S. degree from Hanyang University, Seoul, Korea, in 2000, and his Ph.D. from Texas A\&M University, College Station, TX, in 2003, both in Electrical Engineering. He joined Korea National University of Transportation as professor of the department of Railway Vehicle System Engineering in March 2013. His research interests include motor design, analysis of motor / generator; and applications of motor drive, such as Maglev trains, conventional railway propulsion systems, and modern renewable energy systems.

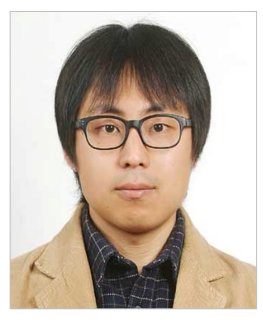

Sungchul Go He received his B.S., M.S. and Ph.D. degrees in Electrical Engineering from Hanyang University, Seoul, Korea in 2004, 2006 and 2010 respectively. He is now working in Samsung Electronics Co. His research interests include design, analysis, testing and control of motor/generator; power conversion systems; and applications of motor drive, 
such as electric vehicles, high-speed maglev train and renewable energy systems.

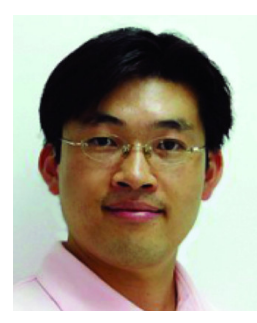

Yonho Cho He received his B.S. and M.S. degrees in Mechanical Engineering from Chonnam University, Gwangju, Korea in 2001 and 2003. He received $\mathrm{Ph} . \mathrm{D}$ degree at University Science and Technology, Daejeon, Korea in 2016. His research interests include analysis and control of dynamic systems of automotive and railway vehicle.

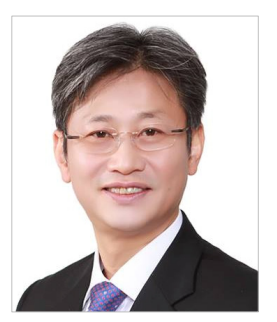

Ju Lee He received his M.S. degree from Hanyang University, Seoul, South Korea, in 1988, and his Ph.D. from Kyusyu University, Japan in 1997, both in Electrical Engineering, He joined Hanyang University in September, 1997 and is currently a Professor of the Division of Electrical and Biomedical Engineering. His main research interests include electric machinery and its drives, electromagnetic field analysis, new transformation systems such as hybrid electric vehicles (HEV), and high-speed electric trains and standardization. $\mathrm{He}$ is a member of the IEEE Industry Applications Society, Magnetics Society, and Power Electronics Society. 